Comment. Math. Helv. 75 (2000) 415-430

(C) 2000 Birkhäuser Verlag, Basel

$0010-2571 / 00 / 030415-15 \$ 1.50+0.20 / 0$

Commentarii Mathematici Helvetici

\title{
Periodic ends, growth rates, Hölder dynamics for automorphisms of free groups
}

\author{
Gilbert Levitt and Martin Lustig
}

\begin{abstract}
Let $F_{n}$ be the free group of rank $n$, and $\partial F_{n}$ its boundary (or space of ends).
For any $\alpha \in$ Aut $F_{n}$, the homeomorphism $\partial \alpha$ induced by $\alpha$ on $\partial F_{n}$ has at least two periodic points of period $\leq 2 n$. Periods of periodic points of $\partial \alpha$ are bounded above by a number $M_{n}$ depending only on $n$, with $\log M_{n} \sim \sqrt{n \log n}$ as $n \rightarrow+\infty$.

Using the canonical Hölder structure on $\partial F_{n}$, we associate an algebraic number $\lambda \geq 1$ to any attracting fixed point $X$ of $\partial \alpha$; if $\lambda>1$, then for any $Y$ close to $X$ the sequence $\partial \alpha^{p}(Y)$ approaches $X$ at about the same speed as $e^{-\lambda^{p}}$. This leads to a set of Hölder exponents $\Lambda_{h}(\Phi) \subset(1,+\infty)$ associated to any $\Phi \in$ Out $F_{n}$. This set coincides with the set of nontrivial exponential growth rates of conjugacy classes of $F_{n}$ under iteration of $\Phi$.
\end{abstract}

Mathematics Subject Classification (2000). 20E05, 20F65.

Keywords. Free group, automorphism, boundary, periodic point, growth rate, Hölder.

\section{Introduction and statement of results}

Let $\varphi$ be a homeomorphism of a closed surface $\Sigma$, with $\chi(\Sigma)<0$. In [14], Nielsen studied $\varphi$ by lifting it to the universal covering $D$ of $\Sigma$ and considering the induced homeomorphism $f$ on the circle at infinity $S$. In more algebraic terms, the mapping class of $\varphi$ corresponds to an outer automorphism $\Phi$ of $\pi_{1} \Sigma$, various lifts of $\varphi$ to $D$ correspond to various automorphisms $\alpha$ of $\pi_{1} \Sigma$ representing $\Phi$, and $f: S \rightarrow S$ corresponds to the homeomorphism $\partial \alpha$ induced by $\alpha$ on the boundary of the group $\pi_{1} \Sigma$.

Let $F_{n}$ be the free group of rank $n$. We will study automorphisms $\alpha$ of $F_{n}$, and outer automorphisms $\Phi \in$ Out $F_{n}$, through the homeomorphisms $\partial \alpha$ induced on the boundary $\partial F_{n}$. The space $\partial F_{n}$, homeomorphic to a Cantor set if $n \geq 2$, may be viewed as the (Gromov) boundary of $F_{n}$, or its space of ends, or the set of right-infinite reduced words in the generators and their inverses.

In the case of a surface group, Nielsen proved among many other things that $f=\partial \alpha: S \rightarrow S$ always has at least two periodic points. Furthermore, the period of these points may be bounded in terms of $|\chi(\Sigma)|$. 
Our first main result has a similar flavor.

Theorem 1. Let $\alpha \in$ Aut $F_{n}$.

(1) The homeomorphism $\partial \alpha: \partial F_{n} \rightarrow \partial F_{n}$ has at least two periodic points of period $\leq 2 n$. If it has only one orbit of periodic points, then this orbit has order two.

(2) Suppose $X \in \partial F_{n}$ is periodic of period $q$ under $\partial \alpha$. Then $q \leq M_{n}$, where $M_{n}$ depends only on $n$ and $\log M_{n} \sim \sqrt{n \log n}$ as $n \rightarrow \infty$.

The bound $2 n$ and the bound on $q$ are sharp. The quantity $\sqrt{n \log n}$ is asymptotic to the logarithm of the maximum order of torsion elements in Aut $F_{n}$, see [11]. As a special case of assertion 2, there is a bound depending only on $n$ for periods of elements $g \in F_{n}$ under the action of $\alpha$. One may also establish a uniform bound for periods of conjugacy classes under the action of $\Phi \in$ Out $F_{n}$. It is proved in [9] that, for "most" $\alpha \in$ Aut $F_{n}$, the homeomorphism $\partial \alpha$ has exactly two fixed points, and no other periodic point.

Like many results of the present paper, the proof of Theorem 1 uses R-trees and techniques introduced in [5]. The proof of assertion 2 uses the main result of [5], and Bestvina-Handel's bound [1] for the rank of the fixed subgroup (the "Scott conjecture").

Let us now consider local properties of fixed points of $\partial \alpha$, using the canonical Hölder structure on $\partial F_{n}$ (see $[3,7]$ ). Let $X$ be a fixed point of $\partial \alpha$ not belonging to the limit set of the fixed subgroup Fix $\alpha \subset F_{n}$. It is either attracting or repelling [5]. In the attracting case, we show that, for $Y \in \partial F_{n}$ close enough to $X$, the sequence $\partial \alpha^{p}(Y)$ converges to $X$ super-exponentially in the sense that

$$
\lim _{p \rightarrow+\infty} \frac{1}{p} \log d\left(\partial \alpha^{p}(Y), X\right)=-\infty,
$$

where $d$ is any distance on $\partial F_{n}$ defining the Hölder structure. We say that $X$ is superattracting (see the beginning of Section 4 for a detailed discussion).

Theorem 2. Let $\alpha \in$ Aut $F_{n}$. Let $X \in \partial F_{n}$ be a superattracting fixed point of $\partial \alpha$. There exists an algebraic number $\lambda=\lambda(\alpha, X) \geq 1$ such that

$$
\lim _{p \rightarrow+\infty} \frac{1}{p} \log \left(-\log d\left(\partial \alpha^{p}(Y), X\right)\right)=\log \lambda
$$

for $Y \in \partial F_{n}$ close to $X$ (and $d$ a distance on $\partial F_{n}$ as above).

Thus, when $\lambda>1$, the sequence $\partial \alpha^{p}(Y)$ converges to $X$ at about the same speed as $f^{p}(x)$ approaches 0 , where $f$ is the map $x \mapsto x^{\lambda}:[0,1) \rightarrow[0,1)$.

Example. Consider $\alpha: F_{2} \rightarrow F_{2}$ given by $\alpha(a)=a b a, \alpha(b)=a b$. The number associated to $X=\lim _{p \rightarrow+\infty} \alpha^{p}(a)=$ ababaaba $\ldots$ is the Perron-Frobenius eigenvalue of the matrix $\left(\begin{array}{ll}2 & 1 \\ 1 & 1\end{array}\right)$. On the other hand, for the polynomially growing 
$\alpha: F_{3} \rightarrow F_{3}$ given by $\alpha(a)=a, \alpha(b)=b a, \alpha(c)=c b a$, the number associated to the superattracting point $X=\lim _{p \rightarrow+\infty} \alpha^{p}(c)=c b a b a^{2} b a^{3} \ldots$ equals 1 .

We now associate a canonical set of Hölder exponents $\Lambda_{h}(\Phi) \subset(1,+\infty)$ to any $\Phi \in$ Out $F_{n}$. View $\Phi$ as a collection of automorphisms $\alpha \in$ Aut $F_{n}$. We say that $\mu>1$ belongs to $\Lambda_{h}(\Phi)$ if there exist $\beta \in \Phi^{q}$, with $q \geq 1$, and a superattracting fixed point $X$ of $\partial \beta$ with $\lambda(\beta, X)=\mu^{q}$. The set $\Lambda_{h}(\Phi)$ is a conjugacy invariant of $\Phi$.

Example. If $\Phi$ is induced by a homeomorphism $\varphi$ of a compact surface $\Sigma$ with $\pi_{1} \Sigma \simeq F_{n}$, then $\Lambda_{h}(\Phi)$ consists of (roots of) the expansion factors of the pseudoAnosov pieces of $\varphi$. They are algebraic units.

If $\alpha \in$ Aut $F_{3}$ is given by $\alpha(a)=a b^{-1}, \alpha(b)=b a c^{-1}, \alpha(c)=c a^{-3}$ (see [6, Example II.7]), then $\Lambda_{h}(\Phi)$ consists of the real root $\lambda$ of $x^{3}-3 x^{2}+2 x-3$. Note that $\lambda$ is not an algebraic unit, and therefore cannot be read off the graph of groups constructed by Sela in Theorem 4.1 of [15].

Theorem 3. Given $\Phi \in$ Out $F_{n}$, the set of Hölder exponents $\Lambda_{h}(\Phi)$ equals the set $\Lambda(\Phi)$ of nontrivial exponential growth rates of conjugacy classes of $F_{n}$ under iteration of $\Phi$.

The (exponential) growth rate of a conjugacy class $\gamma$ is $\lambda(\gamma)=\lim _{p \rightarrow+\infty}\left|\Phi^{p}(\gamma)\right|^{1 / p}$ (see Proposition 3.3). It is nontrivial if $\lambda(\gamma)>1$. It will be shown in [10] that $\Lambda(\Phi)$ has at most $\left[\frac{3 n-2}{4}\right]$ elements and consists of certain Perron-Frobenius eigenvalues of the transition matrix associated to a relative train track representative of $\Phi$.

This paper is organized as follows. In Section 1 we prove the existence of periodic points for $\partial \alpha$. The proof of Theorem 1 is completed in Section 2 (Theorems 2.1 and 2.3). In Section 3 we briefly discuss growth rates. We start Section 4 by a general discussion of superattractivity, valid for an arbitrary hyperbolic group. We then prove Theorem 2.

\section{Existence of periodic points}

Let $F_{n}$ be a free group. We consider its boundary $\partial F_{n}$, equipped with the natural action of $F_{n}$ by left-translations. It is a Cantor set if $n \geq 2$ (it consists of two points if $n=1$ ). In section 4 , we will view $\partial F_{n}$ as a set of right-infinite reduced words. A finitely generated subgroup $J \subset F_{n}$ is quasiconvex [16]. In particular, we can identify the boundary (or limit set) $\partial J$ with a subset of $\partial F_{n}$.

An automorphism $\alpha \in \operatorname{Aut} F_{n}$ is a quasi-isometry of $F_{n}$. It induces a homeomorphism $\partial \alpha: \partial F_{n} \rightarrow \partial F_{n}$, and a homeomorphism $\bar{\alpha}=\alpha \cup \partial \alpha$ on the compact space $\bar{F}_{n}=F_{n} \cup \partial F_{n}$.

The fixed subgroup Fix $\alpha=\left\{g \in F_{n} \mid \alpha(g)=g\right\}$ has finite rank (Gersten, see 
e.g. [2]). Its boundary $\partial(\operatorname{Fix} \alpha)$ is a subspace of $\partial F_{n}$ upon which $\partial \alpha$ acts as the identity. Note that for any integer $q$ the subgroup Fix $\alpha^{q}$ is $\alpha$-invariant (i.e. it is mapped to itself by $\alpha$ ).

Following Nielsen [14], we say that a fixed point $X$ of $\partial \alpha$ is singular if $X \in$ $\partial(\operatorname{Fix} \alpha)$, regular otherwise.

It is shown in [5, Proposition 1.1] that a regular fixed point $X$ of $\partial \alpha$ is either attracting or repelling. Attracting means that $\bar{\alpha}^{p}(Y)$ converges to $X$ for every $Y$ in a neighborhood of $X$ in $F_{n} \cup \partial F_{n}$ (as $p \rightarrow+\infty$ ), repelling means attracting for $\alpha^{-1}$ (see a detailed discussion in Section 4).

We say that $X \in \partial F_{n}$ is periodic if there exists $q \geq 1$ with $\partial \alpha^{q}(X)=X$. The smallest such $q$ is the period of $X$ and the set $\left\{X, \partial \alpha(X), \ldots, \partial \alpha^{q-1}(X)\right\}$ is a periodic orbit of order $q$. We define $X$ to be regular, attracting... if it is as a fixed point of $\partial \alpha^{q}$. We give a similar definition for a periodic orbit, noting that all its elements have the same type.

Theorem 1.1. Let $\alpha \in$ Aut $F_{n}$. The homeomorphism $\partial \alpha: \partial F_{n} \rightarrow \partial F_{n}$ has at least two periodic points. More precisely, either $\partial \alpha$ has at least two periodic orbits, or the unique periodic orbit has order 2 and is the boundary of an $\alpha$-invariant infinite cyclic subgroup.

Example 1.2. We construct a $\partial \alpha$ with only one periodic orbit. First define $\beta: F_{2} \rightarrow F_{2}$ by $a \mapsto a, b \mapsto a b a$. Then $\partial \beta$ has two singular fixed points $a^{ \pm \infty}=$ $\lim _{p \rightarrow+\infty} a^{ \pm p}$. It is easily checked that these are the only periodic points of $\partial \beta$. The automorphism $\beta$ is the square of $\alpha: a \mapsto a^{-1}, b \mapsto a^{-1} b^{-1}$. The map $\partial \alpha$ permutes $a^{\infty}$ and $a^{-\infty}$.

The proof of Theorem 1.1 (to be found below) uses an $\alpha$-invariant $\mathbf{R}$-tree $T$. The main properties of $T$ are summarized as follows.

Theorem 1.3. ([5]) For every automorphism $\alpha$ of $F_{n}$ there exists an $\mathbf{R}$-tree $T$ and a number $\lambda \geq 1$ such that:

(1) $F_{n}$ acts on $T$ non-trivially, minimally, with trivial arc stabilizers.

(2) There exists a homothety $H: T \rightarrow T$ with stretching factor $\lambda$ such that

$$
\alpha(g) H=H g
$$

for all $g \in F_{n}$ (viewing elements of $F_{n}$ as isometries of $T$ ). If $\lambda=1$, then $T$ is simplicial.

(3) There exists an $F_{n}$-equivariant injection $j: \partial T \rightarrow \partial F_{n}$ satisfying $\partial \alpha \circ j=$ $j \circ H$.

Furthermore:

Theorem 1.4. ([6]) Given $Q \in T$, its stabilizer $\operatorname{Stab} Q$ has $r a n k \leq n-1$, and the 
action of $\operatorname{Stab} Q$ on $\pi_{0}(T \backslash\{Q\})$ has at most $2 n$ orbits. The number of $F_{n}$-orbits of branch points is at most $2 n-2$.

A homothety is a map $H$ such that $d(H x, H y)=\lambda d(x, y)$ for some $\lambda>0$ (the stretching factor $)$. We denote $\partial T$ the set of equivalence classes of infinite rays $\rho:(0,+\infty) \rightarrow T$, and again $H: \partial T \rightarrow \partial T$ the induced map. See [5, Sections 2 and 3] for other definitions and a proof of Theorem 1.3. Theorem 1.4 follows from Theorem III.2 of [6]. Given $\alpha$ and $T$, the number $\lambda$ and the homothety $H$ satisfying $\alpha(g) H=H g$ are unique.

A homothety $H$ with $\lambda>1$ has a unique fixed point $Q$, which may be in $T$ or only in its metric completion $\bar{T}$. We define an eigenray of $H$ as in [5], as an isometric map $\rho:(0, \infty) \rightarrow T$ such that $\rho(\lambda x)=H \rho(x)$. We note:

Proposition 1.5. If $H R=R$, the stabilizer Stab $R$ is $\alpha$-invariant. If $\rho$ is an eigenray, then $j(\rho)$ is a fixed point of $\partial \alpha$. Now suppose $\lambda>1$, and let $Q$ be the fixed point of $H$. If $Q \in \bar{T} \backslash T$, then there exists a unique eigenray. If $Q \in T$, then any component of $T \backslash\{Q\}$ that is fixed by $H$ contains a unique eigenray.

Proof of Theorem 1.1. First assume that the fixed subgroup Fix $\alpha^{q}$ is nontrivial for some $q \geq 1$. If it is cyclic, its two boundary points are either fixed points of $\partial \alpha$ or a periodic orbit of order 2. If Fix $\alpha^{q}$ has rank $\geq 2$, we get uncountably many periodic orbits. From now on we assume that $\operatorname{Fix} \alpha^{q}$ is trivial for every $q$, and we construct an attracting periodic orbit of $\partial \alpha$. The same argument, applied to $\alpha^{-1}$, will yield a second orbit.

Let $T$ be as in Theorem 1.3. If $H$ fixes some $Q \in T$ with $\operatorname{Stab} Q$ nontrivial, recall that $\operatorname{Stab} Q$ is $\alpha$-invariant. Since it has rank less that $n$ and $\partial \operatorname{Stab} Q$ embeds into $\partial F_{n}$, we will be able to use induction on $n$ (of course $n=1$ is trivial). Also note that, if $\rho$ is an eigenray of $H$ (with $\lambda>1$ ), then the fixed point $j(\rho)$ of $\partial \alpha$ is attracting (see the proof of Assertion 2 of Proposition 4.4 in [5]).

Recall that we want to find an attracting periodic orbit of $\partial \alpha$. First assume $\lambda>1$. Let $Q \in \bar{T}$ be the fixed point of $H$. If $Q \in \bar{T} \backslash T$, there is an eigenray $\rho$ and $j(\rho)$ is an attracting fixed point of $\partial \alpha$. Suppose $Q \in T$. If $\operatorname{Stab} Q$ is nontrivial, we use induction on $n$. Otherwise $T \backslash\{Q\}$ has at most $2 n$ components by Theorem 1.4, and some power of $H$ has an eigenray. This gives an attracting periodic orbit as before.

Now we assume $\lambda=1$. In this case $T$ is simplicial and $H$ is an isometry.

First suppose $H$ fixes some $Q$. We may assume $\operatorname{Stab} Q$ is trivial (otherwise, we do induction). Then some $H^{k}$ fixes an edge $e$. Replacing $\alpha$ by $\alpha^{k}$, we assume $k=1$. Collapse to a point every edge not in the orbit of $e$ (under the action of $F_{n}$ ). We get a new tree $T^{\prime}$ with an isometry $H^{\prime}$ satisfying the conclusions of Theorem 1.3. The map $H^{\prime}$ fixes some point with nontrivial stabilizer (since all vertices now have nontrivial stabilizer) and we use induction.

The last possibility if that $H$ is a hyperbolic isometry of $T$. In this case $H$ has 
a translation axis $A$ and fixes two ends of $T$. Orienting $A$ by the action of $H$, we consider the positive end $A^{+}$of $A$ and the associated fixed point $X^{+}=j\left(A^{+}\right)$of $\partial \alpha$. We complete the proof by showing that $X^{+}$is not repelling (and therefore is attracting since we assume Fix $\alpha^{q}$ trivial for all $q$ ). Choose any point $Q \in A$, and $g \in F_{n}$ acting on $T$ as a hyperbolic isometry whose axis has compact intersection with $A$. Writing $\alpha^{p}(g) Q=H^{p} g H^{-p} Q$ we see that the projection of $\alpha^{p}(g) Q$ onto $A$ goes to $A^{+}$as $p \rightarrow \infty$. By Section 3 of [5] this implies $\lim _{p \rightarrow \infty} \alpha^{p}(g)=X^{+}$. Thus $X^{+}$cannot be repelling.

\section{Bounding periods}

Theorem 2.1. Let $\alpha \in$ Aut $F_{n}$. Suppose $X \in \partial F_{n}$ is periodic of period $q$ under $\partial \alpha$. Then $q \leq M_{n}$, where $M_{n}$ depends only on $n$ and $\log M_{n} \sim \sqrt{n \log n}$ as $n \rightarrow \infty$.

The quantity $\sqrt{n \log n}$ is Landau's asymptotic estimate for $\log g(n)$, where $g(n)$ is the maximum order of elements in the symmetric group $S_{n}$ [8]. It is shown in [11] that the same estimate holds for the maximum order of torsion elements in $G L(n, \mathbf{Z})$ and Aut $F_{n}$.

We first prove the following special case of Theorem 2.1:

Lemma 2.2. If $g \in F_{n}$ is periodic of period $q$ under $\alpha \in$ Aut $F_{n}$, then $q \leq A_{n}$, where $A_{n}$ is the maximum order of torsion elements in Aut $F_{n}$.

Proof. The subgroup Fix $\alpha^{q}$ is $\alpha$-invariant, and the restriction of $\alpha$ has order exactly $q$ in Aut $\left(\operatorname{Fix} \alpha^{q}\right)$. Since the rank of Fix $\alpha^{q}$ is $\leq n$ by [1], and Aut $F_{k}$ naturally embeds into Aut $F_{\ell}$ for $k<\ell$, the group Aut $F_{n}$ contains an element of order $q$.

Remark. Before the Scott conjecture was proved, Stallings showed [17] that, for a given $\alpha$, there is a bound for periods of elements $g \in F_{n}$.

Proof of Theorem 2.1. Lemma 2.2 shows that singular periodic points of $\partial \alpha$ have period $\leq A_{n}$. Now suppose $X$ is regular, say attracting.

The points $X, \partial \alpha(X), \ldots, \partial \alpha^{q-1}(X)$ are attracting fixed points of $\partial \alpha^{q}$. By Theorem 1 of [5], the action of Fix $\alpha^{q}$ on the set of attracting fixed points of $\partial \alpha^{q}$ has at most $2 n$ orbits. Thus there exist $r \leq 2 n$ and $u \in$ Fix $\alpha^{q}$ such that

$$
\partial \alpha^{r}(X)=u X .
$$

By Lemma 2.2 we have

$$
\alpha^{s}(u)=u
$$

for some $s \leq A_{n}$. 
The above equations yield $\partial \alpha^{r s}(X)=a X$ with

$$
a=\alpha^{(s-1) r}(u) \ldots \alpha^{r}(u) u .
$$

If $a=1$ we get $q \leq r s \leq 2 n A_{n}$. Otherwise we note that $a \in \operatorname{Fix} \alpha^{s}$, and from $X=\partial \alpha^{q r s}(X)=a^{q} X$ we conclude that $X$ is singular, a contradiction.

We have thus shown $q \leq M_{n}=2 n A_{n}$. Since $\log A_{n} \sim \sqrt{n \log n}$ by [11], we have $\log M_{n} \sim \sqrt{n \log n}$.

Remark. The bound $q \leq 2 n A_{n}$ is not quite sharp. But if $\alpha \in$ Aut $F_{n}$ has order $A_{n}$ then generic points of $\partial F_{n}$ have period $A_{n}$ under $\partial \alpha$. Therefore the estimate $\log M_{n} \sim \sqrt{n \log n}$ cannot be improved.

Theorem 2.3. For any $\alpha \in$ Aut $F_{n}$, the map $\partial \alpha: \partial F_{n} \rightarrow \partial F_{n}$ has at least two periodic points of period $\leq 2 n$.

For the automorphism defined by $a_{i} \mapsto a_{i+1}(1 \leq i \leq n-1), a_{n} \mapsto a_{1}^{-1}$, every point of $\partial F_{n}$ has period $2 n$.

Proof. There are two cases. If $\alpha$ has no periodic element $g \neq 1$, then $\partial \alpha$ has at most $2 n$ periodic points of a given type (attracting or repelling) by Theorem 1 of [5]. The other case is taken care of by the following result.

Proposition 2.4. Let $\alpha \in$ Aut $F_{n}$. If there is a nontrivial $\alpha$-periodic element $g \in F_{n}$, then there is one of period $\leq 2 n$.

Proof. Let $q$ be the smallest period of nontrivial periodic elements. Arguing as in the proof of Lemma 2.2, we may assume that $\alpha$ has order $q$. Such an $\alpha$ may be realized as an automorphism of a graph ([4], [18]): there exist a finite graph $\Lambda$, an automorphism $f$ of $\Lambda$ fixing a vertex $v$, and an isomorphism $F_{n} \rightarrow \pi_{1}(\Lambda, v)$ such that the following diagram commutes:

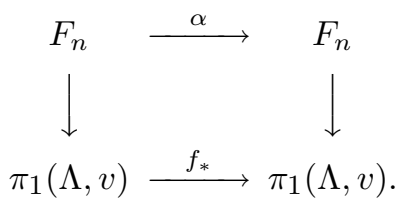

We choose $\Lambda$ with minimal number of vertices. We claim that the action of $\mathbf{Z} / q \mathbf{Z}=\langle f>$ on the set of germs of edges at $v$ is free. This will show $q \leq 2 n$ since $v$ has valence at most $2 n$.

Assume the action is not free. Then some $f^{r}(1 \leq r \leq q-1)$ fixes an edge containing $v$. Let $\Lambda_{0}$ be the component of the fixed point set of $f^{r}$ containing $v$. It is a tree since otherwise $\alpha$ would have a nontrivial periodic element of period $\leq r$. We may therefore collapse $\Lambda_{0}$ to a point, contradicting the choice of $\Lambda$. 


\section{Growth rates}

In this section we fix $\Phi \in$ Out $F_{n}$, and sometimes also an automorphism $\alpha \in \Phi$. We write $|g|$ for the word length of $g \in F_{n}$, and $|\gamma|$ for the length of a conjugacy class $\gamma$ (equal to the length of a cyclically reduced word representing $\gamma$ ).

Let $M$ be the transition matrix of a relative train track map representing $\Phi$ (see [1]). The largest positive eigenvalue (spectral radius) of the matrix $M$ is denoted $\lambda(\Phi)$, or $\lambda(\alpha)$. It is an algebraic integer of degree bounded by $3 n-3$.

For $g \in F_{n}$, the length of $\alpha^{p}(g)$ is bounded from above by a constant times $\|M\|^{p}|g|$. If $\lambda(\Phi)=1$, the growth of $\alpha^{p}(g)$ is polynomial and $\Phi$ is called polynomially growing. For future reference we note:

Remark 3.1. Given $\nu>\lambda(\alpha)$, there exists $C>0$ such that $\left|\alpha^{p}(g)\right| \leq C \nu^{p}|g|$ for all $g \in F_{n}$ and $p \geq 1$.

Now let $\ell: F_{n} \rightarrow \mathbf{R}^{+}$be the length function of an action of $F_{n}$ on an $\mathbf{R}$-tree $T$. It is bounded from above by a constant times word length. In particular, if $T$ is an $\alpha$-invariant $\mathbf{R}$-tree as in Theorem 1.3, we have (up to multiplicative constants) $\lambda^{p} \ell(g)=\ell\left(\alpha^{p}(g)\right) \leq\left|\alpha^{p}(g)\right| \leq\|M\|^{p}|g|$ and therefore $\lambda \leq \lambda(\alpha)$. Conversely:

Proposition 3.2. There exists an $\alpha$-invariant $\mathbf{R}$-tree $T$ as in Theorem 1.3 with $\lambda=\lambda(\alpha)$.

Proof. This is proved by the same arguments as in [5, section 2], but instead of using only the top stratum of the train track (which may lead to $\lambda<\lambda(\alpha)$ ) we use the whole relative train track and an eigenvector $v$ of $M$ associated to $\lambda(\alpha)$. One shows that the resulting action on an $\mathbf{R}$-tree $T$ is nontrivial and has trivial arc stabilizers as in [5]. Minimality of the action may be achieved by restricting to the minimal invariant subtree. It is often more convenient, though, to work with the metric completion $\bar{T}$ of $T$ so as to ensure that $H$ has a fixed point $Q$ when $\lambda(\alpha)>1$.

Now let $J$ be a finitely generated malnormal subgroup of $F_{n}$ (recall that $J$ is malnormal if $\left.g J g^{-1} \cap J \neq\{1\} \Longrightarrow g \in J\right)$. We say that $J$ is $\Phi$-periodic if there exist $q \geq 1$ and $\beta \in \Phi^{q}$ with $\beta(J)=J$. Note that, by malnormality, the class of $\beta$ in Out $J$ is uniquely determined.

For example, suppose that $T$ is an $\mathbf{R}$-tree as in Theorem 1.3 and $J=\operatorname{Stab} Q$ for some branch point $Q$. Then $J$ is malnormal (because arc stabilizers are trivial). By Theorem 1.4, it has rank $<n$. We claim that it is $\Phi$-periodic. Indeed, by Theorem 1.4 there exist $m \in F_{n}$ and $q \geq 1$ such that $m H^{q}$ fixes $Q$. Denoting $i_{m}(g)=m m^{-1}$, the automorphism $\beta=i_{m} \circ \alpha^{q} \in \Phi^{q}$ maps $J$ to itself.

If $J$ is finitely generated, malnormal, $\Phi$-periodic, we define $\lambda_{J}=\lambda\left(\beta_{\mid J}\right)^{\frac{1}{q}}$. 
Proposition 3.3. Let $\Phi \in$ Out $F_{n}$.

(1) Each conjugacy class $\gamma$ in $F_{n}$ has a growth rate $\lambda(\gamma)=\lim _{p \rightarrow+\infty}\left|\Phi^{p}(\gamma)\right|^{1 / p}$.

(2) Given $\lambda \geq 1$, the following are equivalent:

- $\lambda=\lambda(\gamma)$ for some conjugacy class $\gamma$.

- $\lambda=\lambda_{J}$ for some malnormal $\Phi$-periodic subgroup $J \subset F_{n}$ of rank $\leq n$.

The existence of the limit in assertion 1 is folklore (compare [1, Remark 1.8]). Simple examples show that one cannot restrict to free factors in assertion 2.

Proof. The proof is by induction on $n$. Let $T$ be an $\alpha$-invariant $\mathbf{R}$-tree with $\lambda=\lambda(\Phi)$ (see Proposition 3.2). We distinguish two cases, by evaluating the length function on $\gamma$.

If $\ell(\gamma)>0$, we write $\left|\Phi^{p}(\gamma)\right| \geq \ell\left(\Phi^{p}(\gamma)\right)=\lambda^{p} \ell(\gamma)$ (up to a constant) and we conclude that $\gamma$ has growth rate $\lambda(\gamma)=\lambda=\lambda(\Phi)$ (recall that the exponential growth of $\Phi^{p}(\gamma)$ is bounded from above by $\lambda(\Phi)$ ). Note that there exist classes with $\ell(\gamma)>0$, hence there exist classes with growth rate $\lambda(\Phi)$.

If $\ell(\gamma)=0$, an element $g \in F_{n}$ representing $\gamma$ fixes some branch point $Q \in T$, and we argue by induction by considering $\gamma$ as a conjugacy class in $J=\operatorname{Stab} Q$. We have pointed out earlier that $J$ is malnormal, $\Phi$-periodic, of rank $<n$. If $\beta=i_{m} \circ \alpha^{q}$ leaves $J$ invariant, note that, by quasiconvexity of $J$, the growth rate of $\gamma$ under $\beta_{\mid J}$ is the same as the growth rate of $\gamma$, viewed as a conjugacy class in $F_{n}$, under $\Phi^{q}$.

These arguments show that every $\gamma$ has a growth rate, which is of the form $\lambda_{J}$ with $J$ as in the proposition. Conversely, given $J$, let $\ell_{J}$ be the length function of a $\beta_{\mid J}$-invariant tree with $\lambda=\lambda\left(\beta_{\mid J}\right)$. Conjugacy classes with $\ell_{J}(\gamma)>0$ have growth rate $\lambda_{J}$ under $\Phi$.

Definition. We call $\lambda(\Phi)$ the top growth rate of $\Phi$. The set of growth rates $\Lambda(\Phi) \subset(1, \infty)$ consists of the growth rates $\lambda(\gamma)$ which are bigger than 1 .

Note that $\Lambda(\Phi)$ consists of algebraic integers of degree $\leq 3 n-3$, and that $\lambda(\Phi)$ is the largest element of $\Lambda(\Phi) \cup\{1\}$. See [10] for more results about $\Lambda(\Phi)$.

\section{Hölder dynamics}

\section{Superattractivity}

The discussion in this subsection (including Proposition 4.1) is valid for automorphisms of arbitrary (word) hyperbolic groups, but for simplicity we restrict to the case of $F_{n}$ (the generalization is almost immediate using [13]).

Fixing a free basis of $F_{n}$, we may view $\partial F_{n}$ as the set of right-infinite reduced words. Let $X \in \partial F_{n}$ be a fixed point of the homeomorphism $\partial \alpha$ induced by 
$\alpha \in$ Aut $F_{n}$ on $\partial F_{n}$. We say that $X$ is singular if it belongs to the limit set of the fixed subgroup Fix $\alpha$, regular otherwise (recall that Fix $\alpha$ has finite rank).

As explained in [5], there is a basic trichotomy: either $X$ is singular, or $X$ is attracting, or $X$ is repelling (i.e. attracting for $\alpha^{-1}$ ). Attractivity has a strong meaning here (see section 1 of [5]): given $A$, there exists $m$ such that for $Y \in$ $F_{n} \cup \partial F_{n}$

$$
c_{X} Y \geq m \Longrightarrow c_{X}(\partial \alpha(Y))-c_{X} Y>A,
$$

where $c_{X} Y$ is the length of the maximal common initial segment between the reduced words $X$ and $Y$ (i.e. the Gromov scalar product $\langle X, Y\rangle$ with basepoint at the identity in the Cayley graph).

In particular, we have $\lim _{p \rightarrow+\infty} \bar{\alpha}^{p}(Y)=X$ uniformly on a neighborhood of $X$ in $F_{n} \cup \partial F_{n}$ if $X$ is attracting (whereas if $X$ is singular there are fixed points of $\alpha$ in $F_{n}$ arbitrarily close to $X$ ). For the automorphism $\beta$ studied in Example 1.2, the (singular) fixed points $a^{ \pm \infty}$ of $\partial \beta$ are partly repelling and partly attracting: for any $k \in \mathbf{Z}$ we have $\lim _{p \rightarrow+\infty} \partial \beta^{p}\left(a^{k} b Y\right)=a^{\infty}$ if $Y$ is a right-infinite reduced word not starting with $b^{-1}$, but $\lim _{p \rightarrow+\infty} \partial \beta^{p}\left(a^{k} b^{-1} Y\right)=a^{-\infty}$ if $Y$ does not start with $b$.

Also note that an isolated fixed point of $\partial \alpha$ is singular if and only if it belongs to the limit set of an $\alpha$-invariant cyclic subgroup (for the "only if" direction, simply observe that $\alpha$ leaves invariant the stabilizer of $X$ for the action of $F_{n}$ on $\partial F_{n}$ ). In particular, the natural action of Fix $\alpha$ on the set of regular fixed points of $\partial \alpha$ is free. This action has finitely many orbits [2], indeed it follows from [5] that the number of orbits is at most $4 n$. It is not clear to us whether there is a bound depending only on $G$ when $G$ is an arbitrary hyperbolic group.

Now recall that the boundary of $F_{n}$ (of any hyperbolic group, in fact) has a canonical Hölder structure (see [3], [7]). It may be viewed as a collection $\mathcal{D}$ of distance functions on $\partial F_{n}$ that are pairwise bi-Hölder equivalent: Given $d, d^{\prime} \in \mathcal{D}$, there exist $C>0$ and $\beta \in(0,1]$ such that $\frac{1}{C} d^{\frac{1}{\beta}} \leq d^{\prime} \leq C d^{\beta}$. This Hölder structure is preserved by $\partial \alpha$ for every $\alpha \in$ Aut $F_{n}$. If $J \subset F_{n}$ has finite rank, the inclusion $\partial J \hookrightarrow \partial F_{n}$ is bi-Hölder.

We represent the Hölder structure by the visual metrics $d_{\varepsilon}(X, Y)=\exp \left(-\varepsilon c_{X} Y\right)$.

Let $X \in \partial F_{n}$ be a fixed point of $\partial \alpha$, and $d=d_{\varepsilon}$ a visual metric. If $X$ is regular, attracting, it follows from (1) that

$$
\lim _{Y \rightarrow X} \frac{d(\partial \alpha(Y), X)}{d(Y, X)}=0 .
$$

If $X$ is repelling or singular, however, the above quotient is bounded away from 0 on a neighborhood of $X$ (if $X$ is singular, $c_{X}(\partial \alpha(Y))-c_{X} Y$ is bounded near $X$ because Fix $\alpha$ is quasiconvex and $\alpha$ is a quasi-isometry).

Thus (2) is a metric characterization of attracting regular fixed points, similar to the characterization of a superattracting fixed point $c$ of a holomorphic map 
$f: \mathbf{C} \rightarrow \mathbf{C}$ by $f^{\prime}(c)=0$. For this reason, we call an attracting regular point superattracting (and a repelling regular point superrepelling).

Of course the map $\partial \alpha$ is a homeomorphism, and superattracting fixed points may exist only because $\partial \alpha$ is bi-Hölder but in general not bi-Lipschitz. For instance, if $t$ is any lift to the Poincaré disc of a pseudo-Anosov diffeomorphism of a closed hyperbolic surface, then the homeomorphism induced by $t$ on the circle at infinity is never bi-Lipschitz (see Remark (22.14) in [12]).

Characterization (2) above does not depend on the chosen visual metric $d$, but it is not valid for arbitrary metrics in $\mathcal{D}$. The following characterization will apply to every $d \in \mathcal{D}$.

Proposition 4.1. Let $\alpha \in$ Aut $F_{n}$. A fixed point $X$ of $\partial \alpha$ is superattracting if and only if

$$
\lim _{p \rightarrow+\infty} \frac{1}{p} \log d\left(\partial \alpha^{p}(Y), X\right)=-\infty
$$

for $Y \in \partial F_{n}$ close to $X$, where $d$ is any metric on $\partial F_{n}$ defining the Hölder structure.

This equation means that $\partial \alpha^{p}(Y)$ converges to $X$ super-exponentially as $p \rightarrow$ $\infty$. Unlike (2), it is true for every metric in $\mathcal{D}$ if it is true for one.

Proof. We may assume that $d$ is a visual metric. Suppose $X$ is superattracting. We have to prove $\lim _{p \rightarrow \infty} \frac{1}{p} c_{X}\left(\partial \alpha^{p}(Y)\right)=+\infty$ for $Y$ close to $X$. Given $A>0$, let $m$ be as in (1). If $\lim _{p \rightarrow \infty} \partial \alpha^{p}(Y)=X$, there exists $n_{0}$ such that $c_{X}\left(\partial \alpha^{p}(Y)\right) \geq m$ for $p \geq n_{0}$. For $p$ large, we then have

$$
c_{X}\left(\partial \alpha^{p}(Y)\right) \geq A\left(p-n_{0}\right)+m
$$

and the result follows.

Conversely, if $X$ is singular, then $c_{X}(\partial \alpha(Z))-c_{X} Z$ is bounded in a neighborhood of $X$, and therefore $\frac{1}{p} \log d\left(\partial \alpha^{p}(Y), X\right)$ is bounded from below as $p \rightarrow \infty$.

\section{Speed of convergence}

We consider $\alpha \in$ Aut $F_{n}$, and the associated $\Phi \in$ Out $F_{n}$. Recall that $\Lambda(\Phi) \subset$ $(1, \infty)$ is the set of nontrivial growth rates. It may also be viewed as a set of $\lambda_{J}$ (see Proposition 3.3).

Theorem 4.2. Let $\alpha \in$ Aut $F_{n}$. Let $X \in \partial F_{n}$ be a superattracting fixed point of $\partial \alpha$. There exists $\lambda=\lambda(\alpha, X) \in \Lambda(\Phi) \cup\{1\}$ such that

$$
\lim _{p \rightarrow+\infty} \frac{1}{p} \log \left(-\log d\left(\partial \alpha^{p}(Y), X\right)\right)=\log \lambda
$$


for $Y \in \partial F_{n}$ close to $X$ (and any metric d on $\partial F_{n}$ defining the Hölder structure).

Conversely, given $\mu \in \Lambda(\Phi)$, there exist $q \geq 1$, an automorphism $\beta \in \Phi^{q}$, and a superattracting fixed point $X$ of $\partial \beta$ with $\lambda(\beta, X)=\mu^{q}$.

It follows that the set $\Lambda_{h}(\Phi)$ of Hölder exponents defined in the introduction equals $\Lambda(\Phi)$. Note that replacing $d$ by a metric bi-Hölder equivalent to $d$ does not affect the validity of (3).

Proof of Theorem 4.2. We fix a basis of $F_{n}$ and we consider the corresponding Cayley tree $\Gamma$.

Let $X$ be a superattracting fixed point of $\partial \alpha$. We need to prove

$$
\lim _{p \rightarrow+\infty} \frac{1}{p} \log c_{X}\left(\partial \alpha^{p}(Y)\right)=\log \lambda .
$$

We will bound the left-hand side, first from above and then from below.

Lemma 4.3. Suppose $X \in \partial J$, with $J \subset F_{n}$ a finitely generated $\alpha$-invariant malnormal subgroup. Then

$$
\limsup _{p \rightarrow+\infty} \frac{1}{p} \log c_{X}\left(\partial \alpha^{p}(Y)\right) \leq \log \lambda_{J}
$$

for all $Y \in \partial F_{n}$.

Recall that $\lambda_{J}$ is the top growth rate of $\alpha_{\mid J}$.

Proof. Let $x_{t_{p}}$ be the projection of $\partial \alpha^{p}(Y)$ onto the geodesic from $1 \in F_{n}$ to $X$ in $\Gamma$. By quasiconvexity of $J$, we can find $j_{p} \in J$ within a fixed distance of $x_{t_{p}}$. We need to prove $\limsup _{p \rightarrow+\infty} \frac{1}{p} \log \left|j_{p}\right| \leq \log \lambda_{J}$. We will work with word length $\left|j_{p}\right|_{J}$ in $J$, which is comparable to $\left|j_{p}\right|$.

Define $w_{p} \in J$ by $j_{p}=\alpha\left(j_{p-1}\right) w_{p}$. Since $\alpha$ is a quasi-isometry, there is a uniform bound for $\left|w_{p}\right|$, hence also for $\left|w_{p}\right|_{J}$ because $J$ is quasiconvex. Now write

$$
j_{p}=\alpha^{p}\left(j_{0}\right) \alpha^{p-1}\left(w_{1}\right) \cdots \alpha\left(w_{p-1}\right) w_{p} .
$$

For $\nu>\lambda_{J}$ we have

$$
\left|j_{p}\right|_{J} \leq C \nu^{p}\left|j_{0}\right|_{J}+C \nu^{p-1}\left|w_{1}\right|_{J}+\cdots+C \nu\left|w_{p-1}\right|_{J}+\left|w_{p}\right|_{J}
$$

with $C$ given by Remark 3.1. Thus $\left|j_{p}\right|_{J}=O\left(\nu^{p}\right)$ for all $\nu>\lambda_{J}$, and the lemma is proved.

Corollary 4.4. Theorem 4.2 holds if $\alpha$ is polynomially growing (i.e. $\lambda(\alpha)=1$ ), with $\lambda(\alpha, X)=1$. 
Fix a subgroup $J$ as in Lemma 4.3, and consider an R-tree $T$ with an action of $J$ satisfying the conditions of Theorem 1.3 with respect to $\alpha_{\mid J}$. Using Proposition 3.2 , we assume that the stretching factor of the homothety $H$ equals $\lambda_{J}$. Suppose furthermore $\lambda_{J}>1$.

Lemma 4.5. Suppose $X=j(\rho)$, where $\rho$ is an eigenray of $H: T \rightarrow T$ (in particular, $X \in \partial J)$. Then

$$
\liminf _{p \rightarrow+\infty} \frac{1}{p} \log c_{X}\left(\partial \alpha^{p}(Y)\right) \geq \log \lambda_{J}
$$

for $Y \in \partial F_{n}$ close enough to $X$.

Proof. With the notations of Section 1 , let $Q \in \bar{T}$ be the fixed point of $H$ (i.e. the origin of $\rho)$. Choose $j_{p}$ as in the proof of Lemma 4.3 and define $d_{p}$ as $\bar{d}\left(Q, j_{p} Q\right)$ (where $\bar{d}$ denotes the distance in $\bar{T}$ ). Note that

$$
\bar{d}\left(Q, \alpha\left(j_{p}\right) Q\right)=\bar{d}\left(Q, \alpha\left(j_{p}\right) H Q\right)=\bar{d}\left(Q, H j_{p} Q\right)=\lambda_{J} \bar{d}\left(Q, j_{p} Q\right) .
$$

On the other hand, recall that the distance in $J$ from $\alpha\left(j_{p}\right)$ to $j_{p+1}$ is bounded independently of $p$ (and of $Y$ ). Thus we obtain an inequality of the form

$$
d_{p+1} \geq \lambda_{J} d_{p}-A
$$

with $A$ independent of $p$ and $Y$.

If $Y$ is close enough to $X$ in $\partial F_{n}$, then $j_{0}$ is close to $X$ in $J \cup \partial J$, and therefore $d_{0}$ is large (by bounded backtracking, see section 3 of [5]). This implies

$$
\liminf _{p \rightarrow+\infty} \frac{1}{p} \log d_{p} \geq \log \lambda_{J}
$$

Finally, we observe that $d_{p}=\bar{d}\left(Q, j_{p} Q\right)$ is bounded above by a constant times $\left|j_{p}\right|_{J}$, hence by a constant times $\left|j_{p}\right|$.

Now we complete the proof of Theorem 4.2. If $\lambda(\alpha)=1$, then we are done by Corollary 4.4. Assume $\lambda(\alpha)>1$, and consider a tree $T$ as in Proposition 3.2, with stretching factor $\lambda(\alpha)$. If $X=j(\rho)$ as in Lemma 4.5, we are done, with $\lambda=\lambda(\alpha)$. If not, then by Proposition 4.3 of [5] we have $X \in \partial \operatorname{Stab} Q$, where $Q \in T$ is the fixed point of $H$ (recall that points of $\bar{T} \backslash T$ have trivial stabilizer).

The subgroup $\operatorname{Stab} Q$ is $\alpha$-invariant, malnormal, and has rank $<n$ (see section 3). Repeat the argument, working with $\alpha_{\mid \operatorname{Stab} Q}$. After a finite number of steps we find that $X \in \partial J$ (with $J$ invariant, malnormal, of rank $<n$ ), and either $\lambda_{J}=1$ or $X=j(\rho)$. It follows from Lemmas 4.3 and 4.5 that Theorem 4.2 holds, with $\lambda(\alpha, X)=\lambda_{J}$. 
Conversely, consider $\mu \in \Lambda(\Phi)$. First suppose $\mu=\lambda(\alpha)$. Consider an R-tree $T$ as in Theorem 1.3, with $\lambda=\lambda(\alpha)$. By Theorem 1.4 and Proposition 1.5, there exist $m \in F_{n}$ and $q \geq 1$ such that $m H^{q}$ has an eigenray $\rho$. Let $\beta=i_{m} \circ \alpha^{q}$, with $i_{m}(g)=m g m^{-1}$. Then $X=j(\rho)$ is a fixed point of $\partial \beta$, and $\lambda(\beta, X)=\lambda(\beta)=\mu^{q}$.

For arbitrary $\mu=\lambda_{J} \in \Lambda(\Phi)$, let $\alpha^{\prime} \in \Phi^{r}$ leave $J$ invariant. The previous argument yields $\beta \in \Phi^{r q}$ and a fixed point $X$ of $\partial \beta$ in $\partial J$ such that $\lambda\left(\beta_{\mid J}, X\right)=\mu^{r q}$. Since the inclusion $\partial J \hookrightarrow \partial F_{n}$ is bi-Hölder, $\lambda(\beta, X)=\lambda\left(\beta_{\mid J}, X\right)$ has the required form.

\section{Acknowledgments}

We thank V. Shpilrain, whose talk at the 1995 Albany conference started this research.

\section{References}

[1] M. Bestvina, M. Handel, Train tracks for automorphisms of the free group Ann. Math. 135 (1992), 1-51.

[2] D. Cooper, Automorphisms of free groups have finitely generated fixed point sets, Jour. Alg. 111 (1987), 453-456.

[3] M. Coornaert, T. Delzant, A. Papadopoulos, Géométrie et théorie des groupes, Lecture Notes 1441 Springer-Verlag, 1990.

[4] M. Culler, Finite groups of outer automorphisms of a free group, Contemp. Math. 33 (1984), 197-207.

[5] D. Gaboriau, A. Jaeger, G. Levitt, M. Lustig, An index for counting fixed points of automorphisms of free groups, Duke Math. Jour. 93 (1998), 425-452.

[6] D. Gaboriau, G. Levitt, The rank of actions on R-trees, Ann. Sc. ENS 28 (1995), 549-570.

[7] E. Ghys, P. de la Harpe (eds.), Sur les groupes hyperboliques d'après Mikhael Gromov. In: Progress in Mathematics 83, Birkhäuser, 1990.

[8] E. Landau, Über die Maximalordnung der Permutation gegebenen Grades, Archiv der Math. und Phys., Ser. 3, 5 (1903), 92-103.

[9] G. Levitt, M. Lustig, Most automorphisms of a hyperbolic group have very simple dynamics, Ann. Sc. ENS, to appear (available from http://picard.ups-tlse.fr/ levitt/).

[10] G. Levitt, M. Lustig, Growth rates of automorphisms of free groups, in preparation.

11] G. Levitt, J.-L. Nicolas, On the maximum order of torsion elements in $G L(n, \mathbf{Z})$ and Aut $\left(F_{n}\right)$, Jour. Alg. 208 (1998), 630-642.

[12] G. D. Mostow, Strong rigidity of locally symmetric spaces, Ann. Math. Studies 78, Princeton Univ. Press.

[13] W. D. Neumann, The fixed subgroup of an automorphism of a word hyperbolic group is rational, Inv. Math. 110 (1992), 147-150.

[14] J. Nielsen, Untersuchungen zur Topologie der geschlossenen zweiseitigen Flächen, Acta Math. 50 (1927), 189-358; II, Acta Math. 53 (1929), 1-76; III; Acta Math. 58 (1932), 87-167; English transl. in Collected mathematical papers Birkhäuser, 1986.

[15] Z. Sela, The Nielsen-Thurston classification and automorphisms of a free group I, Duke Math. Jour. 84 (1996), 379-397.

[16] H. Short, Quasiconvexity and a theorem of Howson's, pp. 168-176 in Group theory from a geometrical viewpoint (E. Ghys, A. Haefliger, A. Verjovsky, eds.), World Scientific, 1991. 
[17] J. R. Stallings, Finiteness properties of matrix representations, Ann. Math. 124 (1986), $337-346$.

[18] B. Zimmermann, Über Homöomorphismen $n$-dimensionaler Henkelkörper und endliche Erweiterungen von Schottky-Gruppen, Comm. Math. Helv. 56 (1981), 474-486.

Gilbert Levitt

Laboratoire Émile Picard

UMR CNRS 5580

Université Paul Sabatier

F-31062 Toulouse Cedex 4

France

e-mail: levitt@picard.ups-tlse.fr
Martin Lustig

Laboratoire de mathématiques

Université d'Aix-Marseille III

F-13397 Marseille Cedex 20

France

e-mail: Martin.Lustig@math.u-3mrs.fr

(Received: January 20, 1999) 